Annales Geophysicae (2002) 20: 301-309 (c) European Geophysical Society 2002

\title{
The magnetopause shape and location: a comparison of the Interball and Geotail observations with models
}

\author{
J. Šafránková ${ }^{1}$, Z. Němeček ${ }^{1}, \check{S ̆}_{\text {. Dušík }}{ }^{1}$, L. Přech ${ }^{1}$, D. G. Sibeck ${ }^{2}$, and N. N. Borodkova ${ }^{3}$ \\ ${ }^{1}$ Charles University, Faculty of Mathematics and Physics, V Holesovickach 2, 18000 Praha 8, Czech Republic \\ ${ }^{2}$ Applied Physics Laboratory, JHU, Laurel, Maryland, USA \\ ${ }^{3}$ Space Research Institute, Moscow, Russia
}

Received: 29 January 2001 - Revised: 15 October 2001 - Accepted: 23 October 2001

\begin{abstract}
A number of magnetopause models have been developed in the course of last three decades. We have chosen seven of them and tested them using a fresh set of magnetopause crossings observed by Interball-1, Magion-4, and Geotail satellites. The crossings cover the magnetopause from the subsolar region up to near-Earth tail $\left(X_{\mathrm{GSE}} \sim\right.$ $-20 R_{E}$ ) and all geomagnetic latitudes. Our study reveals that (1) the difference between investigated models is smaller than the error of prediction caused by the factors not included in models, (2) the dayside magnetopause is indented in the cusp region, (3) the deepness of the indentation can reach $\sim 4 R_{E}$, and (4) the dimensions of the indentation do not depend on the dipole tilt, whereas its location does.
\end{abstract}

Key words. Magnetopause, magnetospheric physics, solar wind

\section{Introduction}

In magnetospheric physics, it is important to have an accurate model for the determination of the size and shape of the magnetopause. In the absence of solar wind coupling to the magnetosphere, these parameters could be predicted by the dynamic and static pressures of the solar wind and the magnetic pressure of the magnetosphere. Based on this assumption, various models have been developed in the past. The earlier statistical study and following empirical model of the average magnetopause shape and size was carried out by Fairfield (1971). Other empirical models followed; Formisano et al. (1979) adopted Fairfield's approach and used nearly all magnetopause crossings available at that time to develop a new model. Detailed studies of magnetopause processes have shown that dayside reconnection leads to the changes of the magnetopause shape and location. For this reason, Sibeck et al. (1991) fitted magnetopause crossings as either a function of dynamic pressure or as a function of the $B_{Z}$

Correspondence to: J. Šafránková

(jana.safrankova@mff.cuni.cz) component of the interplanetary magnetic field (IMF) and Petrinec et al. (1991) fitted the magnetopause as a function of dynamic pressure for strongly northward and strongly southward IMFs separately.

Recent empirical magnetopause models are already bivariate with respect to both dynamic pressure and IMF $B_{Z}$ (e.g. Roelof and Sibeck, 1993; Petrinec and Russell, 1993, 1996; Kuznetsov and Suvorova, 1996; Shue et al., 1997; and Alexeev et al., 1999). The Howe and Binsack (1972) and Petrinec and Russell (1996) models of the nightside magnetopause use inverse trigonometric functions. The other mentioned models adopted either the general equation of an ellipsoid with two parameters (eccentricity and standoff distance) or the general quadratic equation; Shue et al. (1997) used the standoff distance and the level of tail flaring.

From this short survey, it follows that these models use various functional forms to represent the shape and location of the magnetopause and are usually parametrized by solar wind dynamic pressure and IMF $B_{Z}$. The basic findings of these studies were that the magnetopause scales are roughly with pressure as $p^{-1 / 6}\left(p^{-1 / 6.6}\right.$ in Shue et al., 1997) and that for decreasing IMF $B_{Z}$, the magnetopause displaces inward near the nose and outward down the tail. However, the various models have different ranges of validity (both spatially and in control parameters) because, among other things, the data sets used for their development were different. Moreover, the data sets used for the development of models usually contained a rather small number of high-latitude magnetopause crossings.

Sotirelis and Meng (1999) presented a calculation where the shape of the magnetopause is computed from the requirement that the pressure in the magnetosheath is balanced by magnetic pressure inside the magnetosphere. The authors found changes in the shape of the magnetopause with varying dipole tilt angle. The magnetotail and standoff location shifted vertically, in opposite directions, for nonzero dipole tilt. The vertical offset of the standoff location from the Earth-Sun line varies linearly with dipole tilt angle, reaching $\sim 3 R_{E}$ for maximum of the tilt and having a weak depen- 
dence on solar wind dynamic pressure. The magnetic field model used to obtain the magnetospheric magnetic pressure is a modified version of the T96 model (Tsyganenko, 1996).

Similar shifts were reported by Tsyganenko (1998), who used a new method to model the effects of the planetary dipole tilt and the IMF related twisting of the cross-tail current sheet. He concentrated on a deformation, yielding the observed gradual deflection of the magnetotail away from the equatorial plane of the tilted planar dipole and found that the deformation affects not only the shape of the tail current sheet but the entire magnetosphere, including the magnetopause, which shifts along the $Z$ axis in the same direction and with the same amplitude as the cross-tail current sheet. The author found the Earth's dipole tilt effect upon the position of the magnetotail boundary in the distance range $-40<X_{\mathrm{GSM}}<-20 R_{E}$ but suggests tilt related effects on the shape of the magnetopause for other intervals of $X_{\mathrm{GSM}}$. Using the data from Hawkeye 1 high-latitude, highapogee spacecraft, similar results were reported by Eastman et al. (2000).

Boardsen et al. (2000) prepared a new empirical model for the shape of the near-Earth high-latitude magnetopause which is parametrized by solar wind dynamic pressure, IMF $B_{Z}$, and dipole tilt angle and found that dipole tilt angle and solar wind dynamic pressure are the most significant factors influencing the shape of the high-latitude magnetopause, whereas IMF $B_{Z}$ dependence is separable only after the effects of the pressure and dipole tilt angle are removed. However, this model covers only a high-latitude part of the magnetopause in a limited range of the $X_{\mathrm{GSE}}$ coordinate.

As one can see from our short and non-complete list, the variety of models leads often to confusion for potential users. For this reason, we used a completely new set of the magnetopause crossings and compared their coordinates with predictions of several magnetopause models with motivation to analyze deviations between measurements and models in different ranges of $X_{\mathrm{GSE}}$. The set consists of low-latitude as well as high-latitude crossings and it allows us to determine differences between the low- and high-latitude magnetopause.

\section{Data set and methodology}

The basic data set includes a collection of the magnetopause crossings observed by the Interball-Tail project. Both Magion-4 and Interball-1 satellites were launched into an elongated elliptical orbit with inclination of $63^{\circ}$, apogee of $\sim 195000 \mathrm{~km}\left(\sim 30 R_{E}\right)$, and perigee of $\sim 800 \mathrm{~km}$ $\left(\sim 0,12 R_{E}\right)$. Due to orbital parameters and their temporal evolution, the satellites have scanned a broad range of local times throughout the magnetospheric tail toward the subsolar region. These magnetopause crossings represent $\sim 1800$ crossings of the Interball-1 satellite at low- and high-latitudes from August 1995 to October 1997, and 120 crossings of Magion-4, mainly at low-latitudes. It would be pointed out that both Interball-1 and Magion-4 crossed the high-latitude magnetopause often in a close vicinity of the cusp or in the cusp itself. The seasonal evolution of the cusp location and the evolution of the spacecraft orbits go in the same direction which yields good coverage of the magnetopause near the cusp and a lack of mid- and low-latitude crossings in the subsolar region. To improve the coverage of measurements at these latitudes, we have complemented our observations with the Geotail magnetopause crossings during the same time period (approximately $\sim 1700$ crossings). All crossings were spread from the subsolar region to $X_{\mathrm{GSE}} \approx-20 R_{E}$ and occurred under various upstream conditions: the solar wind velocity varied from 300 to $700 \mathrm{~km} / \mathrm{s}$, density from 1 to $35 \mathrm{~cm}^{-3}$, and Mach number from 4 to 50 . The set includes multiple crossings.

Interball-1 and Magion-4 magnetopause crossings were identified manually on the basis of observations of ion and electron energy spectra (Yermolaev et al., 1997; Sauvaud et al., 1997; Nemecek et al., 1997) and the magnetic field (Nozdrachev et al., 1998). Geotail magnetopause crossings were computed automatically on the basis of the magnetic field changes (Ivchenko et al., 2000). The solar wind and IMF data were taken from WIND that was used as a monitor. The time of propagation of the solar wind features from the WIND position to the location of a magnetopause crossing was computed as a two-step approximation from WIND solar wind velocity measurements. In this approximation, we suppose the solar wind velocity equal to $400 \mathrm{~km} / \mathrm{s}$ and determine the time lag from the difference of spacecraft locations along the $X_{\mathrm{GSE}}$ axis. Then we take the actual velocity measured at the lagged time and compute the new lag. A deceleration of the solar wind in the magnetosheath is neglected because an error caused by this deceleration would be smaller than uncertainties caused by oblique fronts of solar wind disturbances. Values of the solar wind dynamic pressure and IMF data used for the comparison with models were computed as five-minute averages centered around the time estimated as given above.

For our magnetopause crossings, we have computed the predicted magnetopause positions according to following models:

- Formisano et al. (1979) (hereafter referred as F79) use the second-order three-dimensional surface for a fit of the data normalized to the averaged solar wind dynamic pressure, $p_{S W}$.

- Sibeck et al. (1991) (hereafter referred as S91) - use six subsets according to IMF $B_{Z}$ and fitted an ellipsoid of revolution to each subset. The ellipsoid parameters are a function of $p_{S W}$.

- Roelof and Sibeck (1993) (hereafter referred as RS93) - use an ellipsoid of revolution in solar-wind aberrated coordinates and expressed the $p_{S W}$ and $B_{Z}$ dependence of each of the three ellipsoid parameters as a secondorder (6-term) bi-variate expansion in $p_{S W}$ and $B_{Z}$.

- Kuznetsov and Suvorova (1996) (hereafter referred as KS96) - use two paraboloids for the fit of the magne- 
topause surface. These paraboloids intersect for the angle $\theta \sim 30^{\circ}\left(\theta\right.$ is an angle between $X_{\mathrm{GSE}}$ and radius vector of the observed crossing). Fits are computed for two IMF $B_{Z}$ orientations.

- Petrinec and Russell (1996) (hereafter referred as PR96) - this model expanded their previous models (Petrinec et al., 1991; Petrinec and Russell, 1993). The dayside $\left(X_{\mathrm{GSE}}>0\right)$ and nightside $\left(X_{\mathrm{GSE}}<0\right)$ parts are fitted separately in different coordinate systems. They use different functional forms for the expression of coefficients of the fit for different signs of IMF $B_{Z}$.

- Shue et al. (1997) (hereafter referred as S97) - introduce a new functional form which is characterized by two parameters, $r_{o}$ and $\alpha$, representing the standoff distance and the level of tail flaring. Both parameters are a function of IMF $B_{Z}$ and dynamic pressure, $p_{S W}$.

- Alexeev et al. (1999) (hereafter referred as A99) - use a paraboloid of revolution for northward and southward IMF, separately.

We describe the accuracy of prediction by a ratio of the predicted $\left(R_{m o d}\right)$ and observed $\left(R_{o b s}\right)$ distance of the magnetopause from the Earth's center computed for each crossing and each model. The set of $R_{\text {mod }} / R_{o b s}$ ratios for a particular model is then fitted by the Gauss function and two parameters of the fit (half-width and center) are presented in the following tables (for further explanation see, Fig. 1).

The second part of the study is devoted to an analysis of the importance of different parameters on the uncertainty of the prediction. For this study, we have computed the relative deviation, $\Delta$ as:

$\Delta=\frac{R_{m o d}-R_{o b s}}{R_{m o d}}$

and plotted this relative deviation as a function of these parameters.

\section{A comparison of magnetopause models}

Most of the investigated models are best fits of a large number of magnetopause crossings. The various authors limited the validity of their fits to a range of upstream parameters and/or $X_{\mathrm{GSE}}$ coordinates according to the coverage of their data sets, the behaviour of their fits, etc. These validity limits are shown in Table 1. We have sorted our data set in accordance with these constraints in order to check each particular model within the range of its declared validity.

Table 2 shows a comparison of models applied to our data set. The assumption behind the computations presented in Table 2 was that models describe the magnetopause location in GSE coordinates. We should note that the GSM coordinates would be better for a description of the magnetopause position (and they really are, as we will show in Sect. 4) but the tested models are axisymmetric with respect to the $X_{\mathrm{GSE}}$ axis and thus there is no difference between the GSE
Table 1. Ranges of the validity of models

\begin{tabular}{|c|c|c|c|c|c|c|}
\hline \multirow{3}{*}{$\begin{array}{c}\text { Model } \\
\text { Sibeck et al., } 1991\end{array}$} & \multicolumn{6}{|c|}{ Range of } \\
\hline & \multicolumn{2}{|c|}{$\begin{array}{l}\mathrm{IMF} B_{Z} \\
{[\mathrm{nT}]}\end{array}$} & \multicolumn{2}{|c|}{$\begin{array}{c}p_{\mathrm{SW}} \\
{[\mathrm{nPa}]}\end{array}$} & \multicolumn{2}{|c|}{$\begin{array}{l}X_{\mathrm{GSE}} \\
{\left[R_{E}\right]}\end{array}$} \\
\hline & -6 & 6 & 0.54 & 9.9 & -20 & 12 \\
\hline $\begin{array}{l}\text { Petrinec and Russell, } \\
1996\end{array}$ & -15 & 10 & 0.109 & 6 & -20 & 12 \\
\hline Shue et al., 1997 & -18 & 15 & 0.5 & 8.5 & -20 & 12 \\
\hline $\begin{array}{l}\text { Kuznetsov and } \mathrm{Su}- \\
\text { vorova, } 1996\end{array}$ & -8 & - & 0.5 & 25 & -10 & 12 \\
\hline $\begin{array}{l}\text { Roelof and Sibeck, } \\
1993\end{array}$ & -5 & 5 & 0.5 & 8 & -20 & 12 \\
\hline Formisano et al., 1979 & 一 & - & 一 & - & -20 & 12 \\
\hline Alexeev et al., 1999 & 一 & - & - & - & -20 & 12 \\
\hline
\end{tabular}

Table 2. Comparison of models in GSE coordinates

\begin{tabular}{lrrr}
\hline \multicolumn{1}{c}{ Model } & Center & Half-width & $\begin{array}{r}\text { Number } \\
\text { of points }\end{array}$ \\
\hline $\begin{array}{l}\text { Sibeck et al., 1991 } \\
\text { Petrinec and Russell, }\end{array}$ & 1.006 & 0.076 & 2931 \\
$\begin{array}{l}\text { 1996 } \\
\text { Shue et al., 1997 }\end{array}$ & 1.007 & 0.068 & 3147 \\
$\begin{array}{l}\text { Kuznetsov and Su- } \\
\text { vorova, 1996 }\end{array}$ & 0.954 & 0.097 & 3201 \\
$\begin{array}{l}\text { Roelof and Sibeck, } \\
\text { 1993 } 1.007\end{array}$ & 0.075 & 3223 \\
$\begin{array}{l}\text { Formisano et al., 1979 } \\
\text { Alexeev et al., 1999 }\end{array}$ & 0.978 & 0.073 & 3220 \\
\hline
\end{tabular}

and GSM coordinate systems. The only exception is the F79 model but this model is explicitly written in GSE.

As there are two parameters determining the accuracy of prediction, it is hard to say which model is better. We can note that the mean location of the magnetopause is described by a majority of the investigated models with $\pm 3 \%$ error and the half-width of the distribution varies from 6.8 to $9.6 \%$. The narrowest distribution is provided by the PR96 and F79 models. This fact is surprising because the F79 model is not parametrized by IMF $B_{Z}$. The reason is that this model is fully $3-\mathrm{D}$ and accounts for the solar wind aberration caused by the Earth's orbital motion, whereas all other models are axisymmetric, i.e. 2-D in nature. For this reason, we have rotated the coordinate system of the observed crossings in the ecliptic plane to remove this effect. Results of a comparison of models with the observed crossings in aberrated coordinates are shown in Table 3 . One can note an improvement of the prediction. This is valid for all models except F79 as one would expect.

The rotation of the coordinates in the ecliptic plane means that we are using coordinates aligned with averaged solar wind velocity because its $v_{X}$ coordinate dominates and the average values of other two coordinates are near zero. However, we are investigating the magnetopause locations in a 
Table 3. Comparison of models in aberrated coordinates

\begin{tabular}{lrrr}
\hline \multicolumn{1}{c}{ Model } & Center & Half-width & $\begin{array}{r}\text { Number } \\
\text { of points }\end{array}$ \\
\hline $\begin{array}{l}\text { Sibeck et al., 1991 } \\
\text { Petrinec and Russell, }\end{array}$ & 0.995 & 0.078 & 2894 \\
1996 & & 0.061 & 3002 \\
$\begin{array}{l}\text { Shue et al., 1997 } \\
\text { Kuznetsov and Su- }\end{array}$ & 0.999 & 0.073 & 3047 \\
vorova, 1996 Sibeck, & 0.995 & 0.096 & 2256 \\
$\begin{array}{l}\text { Roelof and S996 } \\
\text { 1993 }\end{array}$ & 0.072 & 2775 \\
$\begin{array}{l}\text { Formisano et al., 1979 } \\
\text { Alexeev et al., 1999 }\end{array}$ & 1.088 & 0.071 & 3067 \\
\hline
\end{tabular}

particular place and time and the solar wind velocity affecting the magnetopause location has non-zero $v_{Y}$ and $v_{Z}$ components and they can change the actual magnetopause axis. We have rotated coordinates of the observed crossings into a new coordinate system in which the $v_{Y}$ and $v_{Z}$ velocity components vanished using 3-D WIND solar wind velocity measurements. A comparison of models with observations in this solar wind aligned coordinate system is shown in Table 4. One can see that the accuracy of prediction of almost all models is not better than that in Table 3 .

We assume that the $v_{Y}$ and $v_{Z}$ components of the solar wind velocity are caused mainly by the presence of MHD waves in the flow. As these waves have a non-zero velocity of propagation in the plasma rest frame, we cannot predict the actual value of perpendicular velocity components simply by the time shift of data without an analysis of the propagation of the waves. Moreover, the wave mode can change due to the interaction with the bow shock and thus the perpendicular velocity components in the magnetosheath can differ from those in the solar wind. It means that a rotation to the coordinates aligned with solar wind flow cannot bring a further improvement of the prediction, if a distant solar wind monitor is used. Thus, we neglect the perpendicular velocity components and use the aberrated coordinated throughout the rest of the paper for all models except F79. The aberration is an integral part of the F79 model and thus results of this model are presented in standard GSE coordinates.

The distribution of the $R_{\text {mod }} / R_{\text {obs }}$ ratios for the PR96 model is plotted in the left part of Fig. 1. The parameters of the Gaussian fit plotted by a heavy line are listed in Table 3. The right part shows the position of the observed crossings around the model magnetopause surface. The position of the crossings was normalized with respect to the model. One can note a rather small spread of observations in the subsolar region which increases toward the tail.

In Table 5, we are analyzing several factors in order to find sources of this spread. The comparison of the dayside $\left(X_{\mathrm{GSE}}>0\right)$ and nightside $\left(X_{\mathrm{GSE}}<0\right)$ parts shows that both S97 and PR96 models reproduce the mean shape of the magnetopause well but the uncertainty of S97 is higher in the
Table 4. Comparison of models in solar wind aligned coordinates

\begin{tabular}{|c|c|c|c|}
\hline Model & Center & Half-width & $\begin{array}{l}\text { Number } \\
\text { of points }\end{array}$ \\
\hline Sibeck et al., 1991 & 1.000 & 0.077 & 2888 \\
\hline $\begin{array}{l}\text { Petrinec and Russell, } \\
1996\end{array}$ & 1.009 & 0.063 & 2990 \\
\hline Shue et al., 1997 & 1.002 & 0.074 & 3035 \\
\hline $\begin{array}{l}\text { Kuznetsov and } \mathrm{Su}- \\
\text { vorova, } 1996\end{array}$ & 0.946 & 0.100 & 2274 \\
\hline $\begin{array}{l}\text { Roelof and Sibeck, } \\
1993\end{array}$ & 0.999 & 0.074 & 2766 \\
\hline Formisano et al., 1979 & 0.974 & 0.089 & 3055 \\
\hline Alexeev et al., 1999 & 1.090 & 0.086 & 3030 \\
\hline
\end{tabular}

nightside part. On the other hand, the nightside part is described better by S91 and RS93 models.

The magnetopause does not exhibit a significant dawndusk asymmetry in aberrated coordinates because the distributions of the $R_{\text {mod }} / R_{o b s}$ ratios peak at nearly the same values for dawn and dusk crossings (RS93, PR96, S97). The aberration effect is well described by the model surface in F79 but this model places the magnetopause slightly farther from the Earth. The comparison of high and low-latitude crossings suggests an elliptic cross section of the magnetopause, being on average $\sim 5 \%$ flatter in the north-south direction. The same effect was analyzed in Sibeck et al. (1991) with a similar result but it has not been incorporated into their model. The F79 model includes but underestimates the mentioned influence.

To analyze the influence of the upstream parameters, we have sorted out subsets of 500 crossings with lowest/highest solar wind dynamic pressure (lowest in the range of 0.4$1.4 \mathrm{nPa}$ and highest in the range of 3-6 $\mathrm{nPa}$ ) and with highest/lowest IMF $B_{Z}$ values. A comparison of the $\mathrm{S} 97$ model with others (e.g. PR96 or S91) reveals that $\left(p_{S W}\right)^{1 / 6}$ probably better describes the influence of the solar wind dynamic pressure than $\left(p_{S W}\right)^{1 / 6.6}$ used in S97. All models except A99 slightly underestimate the influence of IMF $B_{Z}$ on the magnetopause location; a best fit of this influence seems to be included in PR96.

In order to search for possible sources of uncertainties of predictions of different models, we have plotted results the same way as it is shown in Fig. 1 for the PR96 model. After an analysis of such plots (not presented), we can note that slightly better results provided by the PR96 model are caused by the fact that this model uses two different surfaces for the dayside and nightside magnetopause. The magnetopause shape is probably too complicated to be described by a simple second order surface. The KS96 model uses two different surfaces too, but this model was developed for extreme high solar wind pressure and we have only a few such crossings in our data set.

As we noted above, the PR96 model provides the best description of the magnetopause surface. However, Fig. 1 

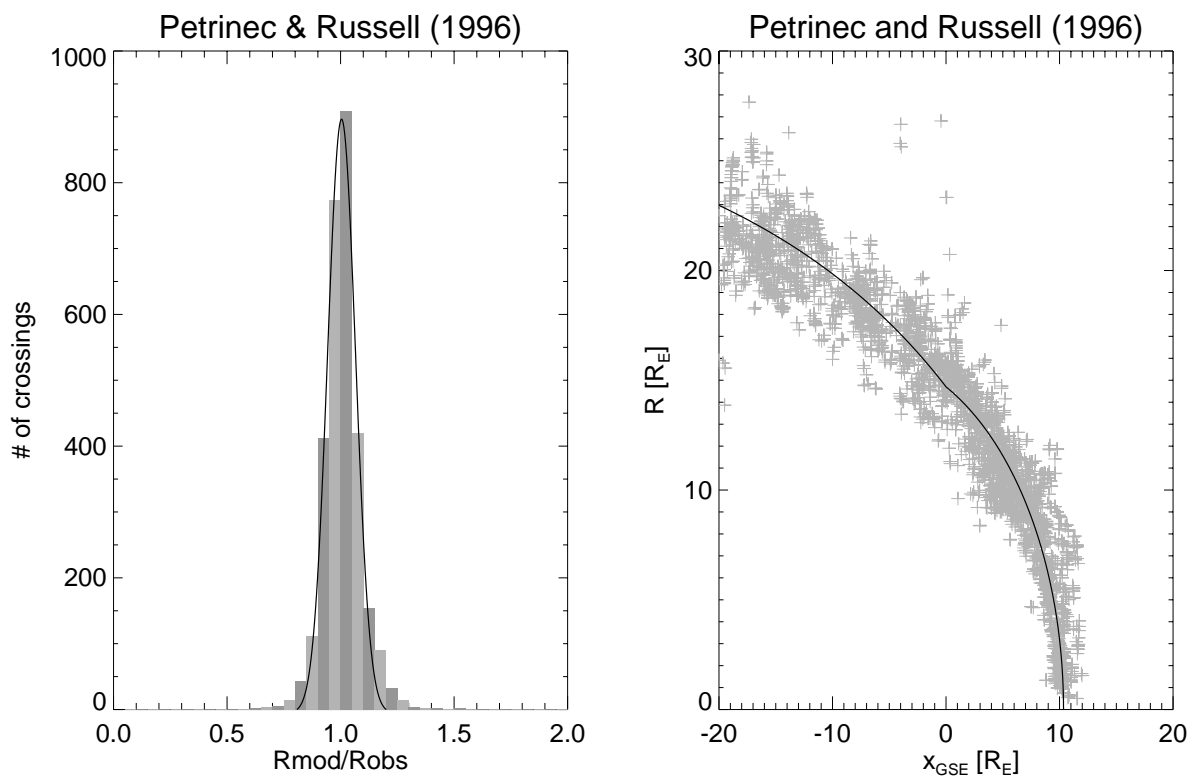

Fig. 1. Histogram of $R_{\text {mod }} / R_{\text {obs }}$ ratios for the PR96 model (left part) and the position of the observed magnetopause crossings in aberrated coordinates scaled to the solar wind dynamic pressure and IMF $B_{Z}$ (right part). The heavy line represents the PR96 magnetopause under standard conditions $\left(p_{S W}=2 \mathrm{nPa}, \mathrm{IMF} B_{Z}=0\right)$.

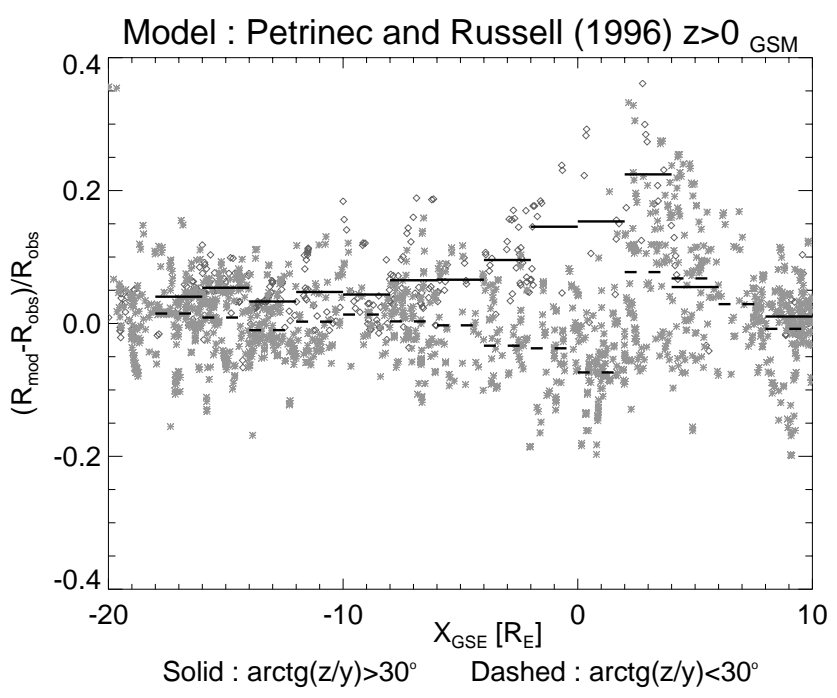

Fig. 2. Distribution of relative deviations of the observed magnetopause crossings from the PR96 prediction along aberrated $X_{\mathrm{GSE}}$ axis. Circles and the heavy line refer to high geomagnetic latitudes.

shows that the spread of observed crossings is rather high and many crossings are observed 2 or $3 R_{E}$ inside or outside the model magnetopause surface.

\section{The high-latitude magnetopause}

As a next step, we have sorted the relative deviation, $\Delta$ according to different parameters and plotted it as a function of $X_{\mathrm{GSE}}$-coordinates. We have concentrated on the Northern Hemisphere $\left(Z_{\mathrm{GSM}} \geq 0\right)$ because our data set covers better this hemisphere and the situation in both hemispheres can be different. Since our results in Table 5 suggest a change in the magnetopause shape with latitude, we have divided our data set into two groups: low-latitude part $\left(\arctan Z_{\mathrm{GSM}} / Y_{\mathrm{GSM}}<\right.$ $\left.30^{\circ}\right)$ and high-latitude part $\left(\arctan Z_{\mathrm{GSM}} / Y_{\mathrm{GSM}}>30^{\circ}\right)$.

Figure 2 depicts a comparison of the high- and low-latitude groups. We can point out that in the range of $\left(X_{\mathrm{GSE}} \leq 3\right)$, the mean values of $\Delta$ for low-latitude crossings (dashed line) lie near zero and below that for high-latitudes. Taking into account a definition of $\Delta$, we can conclude that the PR96 model well describes the shape of the nightside low-latitude magnetopause, whereas the high-latitude magnetopause lies on average inside the predicted position. The difference is $\sim 3-15 \%$ with a trend to decrease down the tail. At the subsolar region $\left(X_{\mathrm{GSE}} \geq 5\right)$, the difference between lowand high-latitudes seems to be negligible.

The most pronounced difference between high- and lowlatitudes is observed in the region $\left(-5 \leq X_{\mathrm{GSE}} \leq 5\right)$. The low-latitude crossings decline from prediction by $\sim 7 \%$ on average (dashed line) but the high-latitude crossings are systematically observed nearer to the Earth than predicted. The peak of $\Delta$ is $\sim 22 \%$ at $X_{\mathrm{GSE}}=+3$

The greatest deviation of the prediction from observations is seen near $X_{\mathrm{GSE}} \sim 0$ where the functional form of PR96 changes and we suppose that this fact could influence the results. For this reason, Fig. 3 shows the same plot as Fig. 2 for S97 which uses the same function throughout the whole range of $X_{\mathrm{GSE}}$. However, we can note similar behaviour for $\Delta$ at high-latitudes. We tested all models and the results were qualitatively similar, and thus we have chosen the PR96 model for further demonstration. The location of the region with greatest deviation of observations from the model 


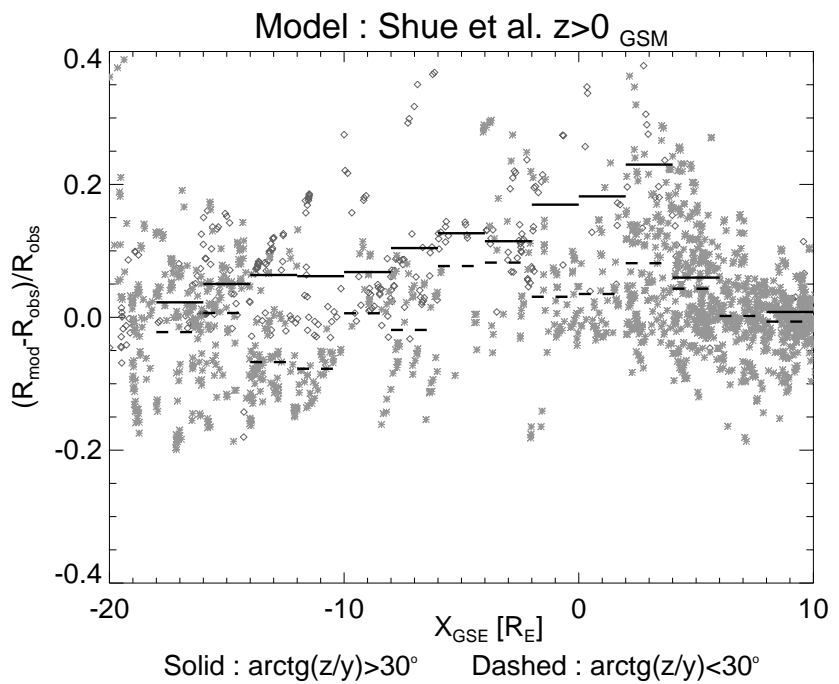

Fig. 3. The same as Fig. 2 for the S97 model.

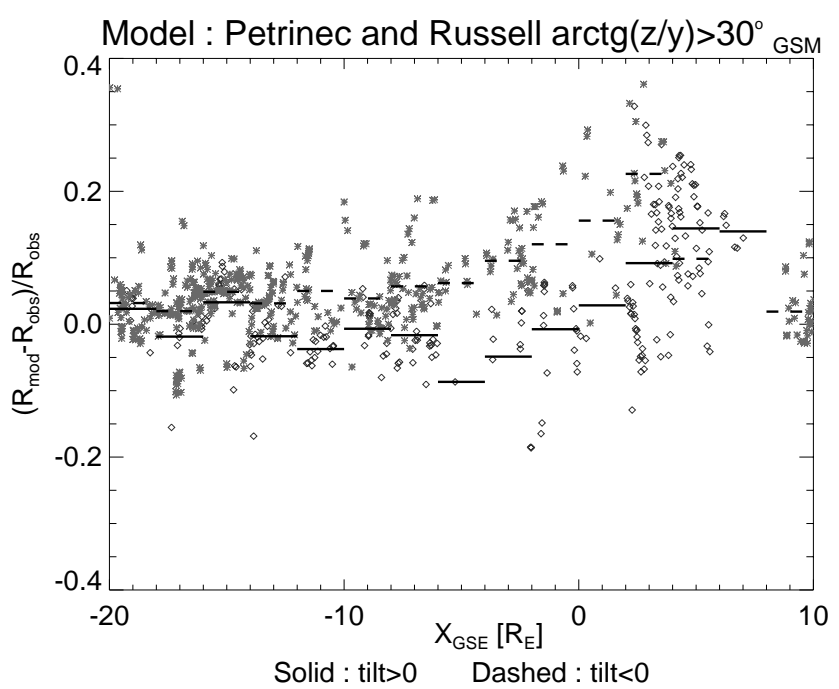

Fig. 4. Comparison of the magnetopause locations for positive (solid line, circles) and negative (dashed line, asterisks) tilt angles at high latitudes.

in high-latitudes and in a limited range of $X_{\mathrm{GSE}}$ suggests that the magnetopause is indented due to the presence of the cusp. This suggestion confirms Fig. 4 where the high-latitude data are plotted for positive and negative tilts. The averaged values show that the indented region shifts sunward for positive tilt as one would expect, if the cusp is a source.

Earlier studies of the cusp precipitation reveal an equatorward shift of the cusp region when IMF $B_{Z}$ becomes negative. Figure 5 shows a comparison of the magnetopause locations for different IMF $B_{Z}$ orientation. It is hard to say if the dependence in Fig. 5 confirms this effect for the magnetopause because our data set suffers from poor data coverage in the range $0<X_{\mathrm{GSE}}<2$. We can note that during intervals of negative IMF $B_{Z}$ the indentation either shifts more sunward or becomes broader.

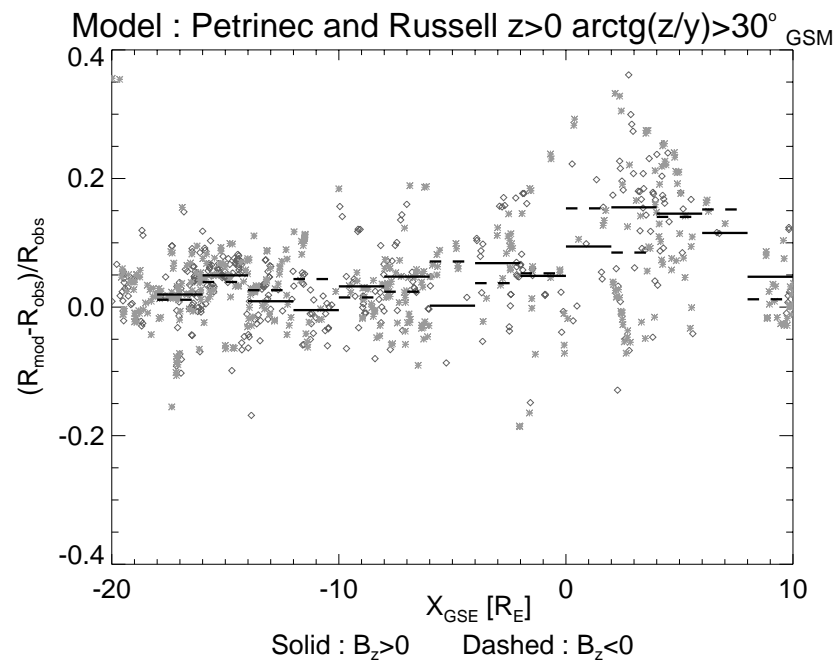

Fig. 5. The same data as in Fig. 4 but sorted according to IMF $B_{Z}$.

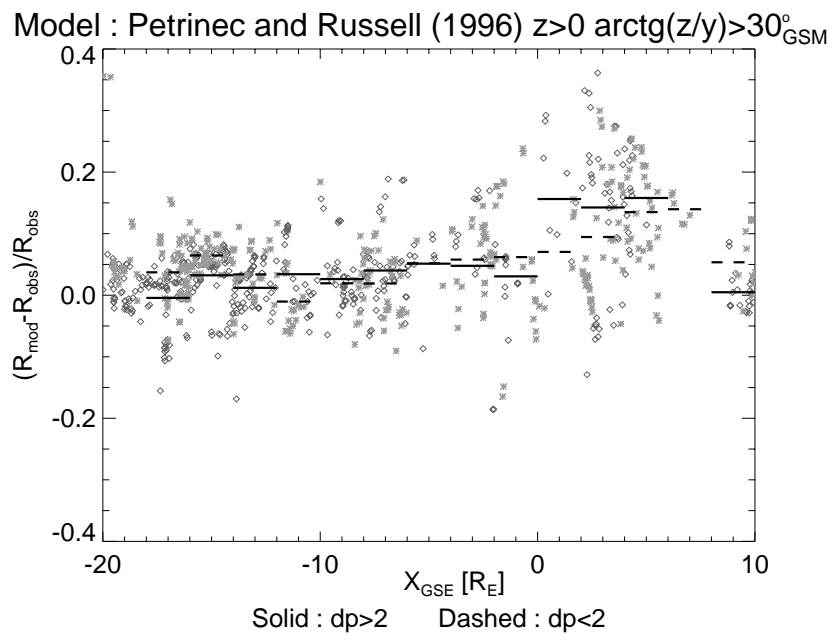

Fig. 6. The same data as in Fig. 4 but sorted according to solar wind dynamic pressure.

Figure 6 shows the high-latitude crossings sorted according to solar wind dynamic pressure. The averaged $\Delta$ does not exhibit any systematic dependence on the nightside magnetopause $\left(X_{\mathrm{GSE}}<0\right)$. However, on the dayside $\left(0 \leq X_{\mathrm{GSE}} \leq\right.$ 6 ), the solar wind pressure effect seems to be stronger than the model predicts because the crossings observed under higher pressure (heavy line) exhibit higher $\Delta$. However, the number of points is rather low and the difference lies in the range of most probable error.

We have analyzed the low-latitude crossings the same way but we did not find any notable change of the profile of $\Delta$ with solar wind dynamic pressure, tilt angle, or IMF $B_{Z}$.

A view on the extent of the cusp indentation is shown in Fig. 7. The coordinates of crossings were normalized to the 
Table 5. Comparison of models under different constraints ( $X, Y, Z$ - aberrated coordinates; $\mathrm{C}$ - center of distribution; HW - half-width of distribution of $R_{\mathrm{mod}} / R_{\mathrm{obs}}$ ratios)

\begin{tabular}{|c|c|c|c|c|c|c|c|c|c|c|c|c|c|c|c|c|c|c|c|c|}
\hline \multirow[t]{3}{*}{ Model } & \multicolumn{20}{|c|}{ Conditions } \\
\hline & \multicolumn{2}{|c|}{$X>0$} & \multicolumn{2}{|c|}{$X<0$} & \multicolumn{2}{|c|}{$Y>0$} & \multicolumn{2}{|c|}{$Y<0$} & \multicolumn{2}{|c|}{ low lat } & \multicolumn{2}{|c|}{ high lat } & \multicolumn{2}{|c|}{$B_{Z(\min )}$} & \multicolumn{2}{|c|}{$B_{Z(\max )}$} & \multicolumn{2}{|c|}{$p_{S W(\min )}$} & \multicolumn{2}{|c|}{$p_{S W(\max )}$} \\
\hline & $\mathrm{C}$ & HW & $\mathrm{C}$ & HW & $\mathrm{C}$ & $\mathrm{HW}$ & $\mathrm{C}$ & HW & $\mathrm{C}$ & HW & $\mathrm{C}$ & HW & $\mathrm{C}$ & HW & $\mathrm{C}$ & HW & $\mathrm{C}$ & HW & $\mathrm{C}$ & HW \\
\hline PR96 & 0.999 & 0.063 & 1.009 & 0.059 & 1.006 & 0.062 & 1.003 & 0.061 & 0.991 & 0.061 & 1.028 & 0.053 & 1.008 & 0.071 & 1.000 & 0.062 & 1.017 & 0.070 & 1.002 & 0.063 \\
\hline S97 & 0.998 & 0.058 & 1.000 & 0.092 & 1.003 & 0.072 & 0.996 & 0.073 & 0.986 & 0.064 & 1.034 & 0.078 & 1.007 & 0.089 & 0.989 & 0.084 & 0.991 & 0.078 & 1.020 & 0.080 \\
\hline KS96 & 0.951 & 0.104 & 0.938 & 0.080 & 0.960 & 0.102 & 0.937 & 0.091 & 0.933 & 0.090 & 0.993 & 0.101 & 0.903 & 0.098 & 0.980 & 0.078 & 0.936 & 0.089 & 0.950 & 0.103 \\
\hline A99 & 1.068 & 0.083 & 1.107 & 0.073 & 1.096 & 0.087 & 1.083 & 0.078 & 1.069 & 0.074 & 1.131 & 0.068 & 1.013 & 0.088 & 1.133 & 0.61 & 1.083 & 0.088 & 1.096 & 0.102 \\
\hline
\end{tabular}
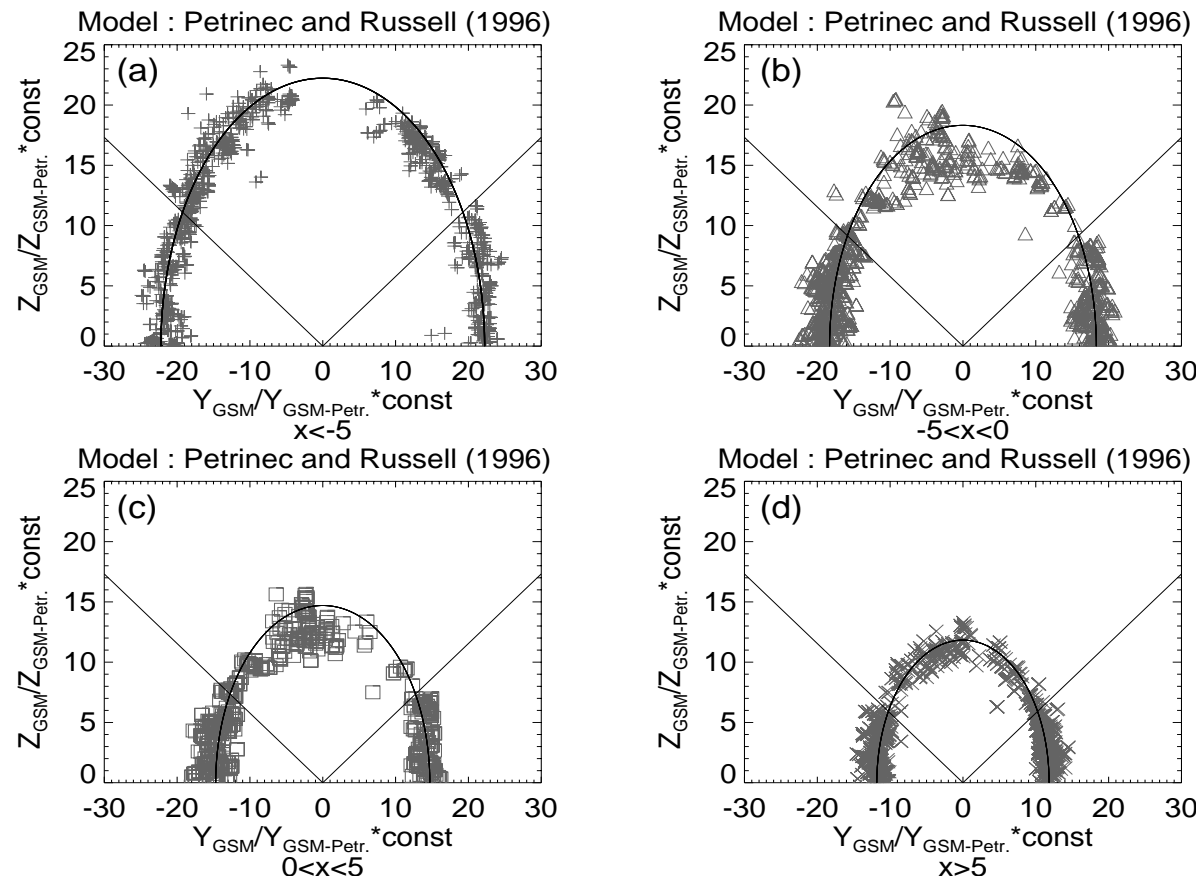

Fig. 7. Projection of the observed magnetopause crossings along the model magnetopause surface (the PR96 model). The oblique straight lines indicate $30^{\circ}$ of geomagnetic latitude. This angle is used as a breakpoint between low- and high-latitudes.

PR96 model:

$Y_{n}=\frac{Y_{\text {obs }}}{Y_{\text {mod }}} \cdot Y_{\text {mod }}\left(X_{n}\right)$

$Z_{n}=\frac{Z_{\text {obs }}}{Z_{\text {mod }}} \cdot Z_{\text {mod }}\left(X_{n}\right)$,

where $Y, Z$ are expressed in GSM coordinates and $X_{n}$ acquires values $-10,-5,0$, and $+5 R_{E}$ for different panels. This normalization projects all points of the model surface onto the heavy line depicted in the panels. The oblique straight lines indicate $30^{\circ}$ of geomagnetic latitude. This angle was used as a breakpoint between low- and high-latitudes in Figs. 2-6. The experimental points are spread around this line symmetrically in low-latitudes in all panels of Fig. 7. A similar conclusion is valid for high-latitude parts of the (a) and (d) panels, whereas nearly all high-latitude crossings lie below the line in the (b) and (c) panels. This comparison of panels shows that the indentation of the magnetopause can be expected in the range from $X_{\mathrm{GSE}}=-5$ to $X_{\mathrm{GSE}}=+5 R_{E}$ at magnetic latitudes higher than $30^{\circ}$ (straight thin lines) and its deepness can reach $\sim 4 R_{E}$.

\section{Summary and conclusion}

We have prepared a fresh set of the low- and high-latitude magnetopause crossings from Interball and Geotail observations and complemented these data with 5-minute averages of the WIND solar wind and IMF measurements. An advantage of this set is that the magnetopause crossings as well 
as the upstream conditions were determined using the same methodology and the same upstream parameter monitor.

This set has been used for the comparison of various magnetopause models. However, our set contains only a few crossings observed under extreme conditions. For this reason, we have not tested the models dedicated predominantly to these conditions, e.g. Shue et al. (1998); Kuznetsov and Suvorova (1998); or Kawano et al. (1999). Moreover, according to Shue et al. (1998, 2000); Kuznetsov and Suvorova (1998), and Petrinec and Russell (1996), the models have almost the same performance during normal solar wind conditions.

We can summarize our investigation as follows:

1. The "aberration" of the solar wind caused by the Earth's orbital motion has a significant effect on the magnetopause location. On the other hand, the influence of the perpendicular components of the solar wind velocity remains under question. A more sophisticated method of the propagation of observations of a distant solar wind monitor toward the Earth is required in order to confirm or exclude this effect. This partial conclusion is in agreement with the note in Boardsen et al. (2000).

2. From a general point of view, the PR96, RS93, S91, and $\mathrm{S} 97$ models are close to each other in our ranges of coordinates $\left(-20 R_{E} \leq X_{\mathrm{GSE}} \leq 12 R_{E}\right)$ and upstream conditions $\left(0.5 \mathrm{nPa} \leq p_{S W}<6 \mathrm{nPa},-7 \mathrm{nT} \leq\right.$ $\left.B_{Z} \leq+9 \mathrm{nT}\right)$. Taking into account both parameters of the Gaussian fits (center and half-width), the PR96 model provides the best prediction but the differences between the aforementioned models are small. In general, the difference among their predictions is significantly smaller than the spread of observations caused by factors which are not included in the models. However, this conclusion cannot be applied to any particular crossing.

3. The $\left(p_{S W}\right)^{1 / 6}$ term probably describes the influence of the solar wind dynamic pressure on the magnetopause location better than $\left(p_{S W}\right)^{1 / 6.6}$ used in S97.

4. All investigated models except A99 slightly underestimate the IMF $B_{Z}$ effect. This is true for positive as well as for negative $B_{Z}$. However, the accuracy of the predictions of the majority of models is better for southward IMF.

5. If the effect of the aberration is removed, the magnetopause does not exhibit any notable dawn-dusk asymmetry. The difference between both flanks is about $1 \%$, comparable to its uncertainty, and thus it is in a range of the most probable error.

6. The high-latitude magnetopause cross section is flattened. The location of this depression is controlled by the tilt angle of the Earth's dipole. A most probable source of the depression is the magnetospheric cusp. Our data show a similar depression for both signs of the tilt, whereas Eastman et al. (2000) noted that when the dipole tilts away from the Sun, the indentation is reduced. The problem of the cusp indentation has a long history and its presence was periodically suggested and then rejected. We assume that the indentation is narrow and its location varies with dipole tilt and upstream conditions and thus this region can be crossed by a spacecraft only occasionally. Interball-1 was launched into the cusp region and thus its coverage of the cusp indentation for negative tilts is probably better than that of Hawkeye.

7. The indentation can be observed at geomagnetic latitudes higher than $30^{\circ}$ and in a broad range of $X_{\mathrm{GSE}}$ coordinates $\left(-2 R_{E} \leq X_{\mathrm{GSE}} \leq 8 R_{E}\right)$. An averaged deepness is $\sim 2.5 R_{E}$ but the magnetopause was often observed $\sim 4 R_{E}$ below the expected location (according to PR96).

8. The position and deepness of the depression is in qualitative agreement with the Sotirelis and Meng (1999) model and with the study of the magnetopause cross section in Sibeck et al. (1991).

9. The location and/or extent of the indentation seems to be controlled by the IMF $B_{Z}$ component.

10. The indented part of the magnetopause seems to be more sensitive to the changes in the solar wind dynamic pressure than other parts.

In order to be "user friendly", the investigated models describe the magnetopause with a simple second-order surface. Such a surface cannot reflect the observed indentation. It results in the fact that models put the low-latitude magnetopause at $1-3 \%$ nearer to the Earth than it is observed. The differences between models can be caused by a number of magnetopause crossings through the indented region used for the development of a particular model.

Acknowledgement. This work was supported by the Czech Grant Agency under Contracts 205/99/1712 and 205/00/1686 and by the Grant Agency of Charles University under Contract 181. Their financial support is greatly acknowledged. Authors are grateful to A. Lazarus and R. Lepping for the WIND plasma and magnetic field data and to L. Frank for the Geotail plasma data.

The authors thank both referees for their assistance in evaluating this paper.

Topical Editor G. Chanteur thanks two references for their help in evaluating this paper.

\section{References}

Alexeev, I. K., Kalgaev, V. V., and Lyutov, Yu. G.: The parabolic magnetopause form and location versus solar wind pressure and IMF, 9th Scientific Assembly of IAGA, Birmingham, A343, 1924 July, 1999.

Boardsen, S. A., Eastman, T. E., Sotirelis, T., and Green, J. L.: An empirical model of the high-latitude magnetopause, J. Geophys. Res., 105, 23 193, 2000. 
Eastman, T. E., Boardsen, S. A., Chen, S.-H., Fung, S. F., and Kessel, R. L.: Configuration of high-latitude and high-altitude boundary layers, J. Geophys. Res., 105, 23 221, 2000.

Fairfield, D. H.: Average and unusual locations of the Earth's magnetopause and bow shock, J. Geophys. Res., 76, 6700, 1971.

Formisano, V., Domingo, V., and Wenzel, K.-P.: The threedimensional shape of the magnetopause, Planet. Space Sci., 27, 1137, 1979.

Howe, H. C. and Binsack, J. H.: Explorer 33 and 35 plasma observations of magnetosheath flow, J. Geophys. Res., 77, 3334, 1972.

Ivchenko, N. V., Sibeck, D. G., Takahashi, K., and Kokubun S.: A statistical study of the magnetosphere boundary crossings by the Geotail satellite, Geophys. Res. Lett., 27, 2881-2884, 2000.

Kawano, H., Petrinec, S. M., Russell, C. T., and Higuchi, T.: Magnetopause shape determinations from measured position and estimated flaring angle, J. Geophys. Res., 104, 247, 1999.

Kuznetsov, S. N. and Suvorova, A. V.: Empirical model of the dayside magnetopause, INP MSU Preprint, 96-37/444, Moskva, 1996.

Kuznetsov, S. N. and Suvorova, A. V.: An empirical model of the magnetopause for broad ranges of solar wind pressure and $B_{z}$ IMF, in: Polar Cap Boundary Phenomena, (Eds) Moen, J., et al., p. 51, Kluwer Acad., Norwell, Mass., USA, 1998.

Nemecek, Z., Fedorov, A., Safrankova, J., and Zastenker, G.: Structure of the low-latitude magnetopause: Magion-4 observations, Ann. Geophysicae, 15, 553, 1997.

Nozdrachev, M. N., Skalsky, A. A., Styazhkin, V. A., and Petrov, V. G.: Some results of magnetic field measurements by the FM-3I flux-gate instrument onboard the Interball-1 spacecraft, Cosmic Research, 36, 268-272, 1998.

Petrinec, S. M., Song, P., and Russell, C. T.: Solar cycle variations in the size and shape of the magnetopause, J. Geophys. Res., 96, 7893, 1991.

Petrinec, S. M. and Russell, C. T.: Factors which control the size of the magnetosphere, in: Solar Terrestrial Predictions IV, vol. 2, (Eds) Hruska, J., et al., pp. 627-635, Natl. Oceanic and Atmos. Admin. Environ. Res. Lab., Boulder, Colo., 1993.

Petrinec, S. M. and Russell, C. T.: Near-Earth magnetopause shape and size as determined from the magnetopause flaring angle, J. Geophys. Res., 101, 137, 1996.

Roelof, E. C. and Sibeck, D. G.: Magnetopause shape as a bivariate function of interplanetary magnetic field $B_{z}$ and solar wind dynamic pressure, J. Geophys. Res., 98, 21 421, 1993.

Sauvaud, J.-A., Koperski, P., Beutier, T., Barthe, H., Aoustin, C., Thocaven, J. J., Rouzaud, J., Penou, E., Vaisberg, O., and Borodkova, N.: The Interball-Tail ELECTRON experiment: initial results on the low-latitude boundary layer of the dawn magnetosphere, Ann. Geophysicae, 15, 587, 1997.

Shue, J.-H., Chao, J. K., Fu, H. C., Khurana, K. K., Russell, C. T., Singer, H. J., and Song, P.: A new functional form to study the solar wind control of the magnetopause size and shape, J. Geophys. Res., 102, 9497, 1997.

Shue, J.-H., Chao, J. K., Fu, H. C., Khurana, K. K., Russell, C. T., Singer, H. J., and Song, P.: Magnetopause location under extreme solar wind conditions, J. Geophys. Res., 103, 17 691, 1998.

Shue, J.-H., Song, P., Russell, C. T., Chao, J. K., and Yang, Y.H.: Toward predicting the position of the magnetopause within geosynchronous orbit, J. Geophys. Res., 105, 2641, 2000.

Sibeck, D. G., Lopez, R. E., and Roelof, E. C.: Solar wind control of the magnetopause shape, location, and motion, J. Geophys. Res., 96, 5489, 1991.

Sotirelis, T. and Meng, C.-I.: Magnetopause from pressure balance, J. Geophys. Res., 104, 6889, 1999.

Tsyganenko, N. A.: Effects of the solar wind conditions on the global magnetospheric configuration as deduced from data-based field models, in: Proceeding of Third International Conference on Substorms (ICS-3), Eur. Space Agendcy Spec. Publ., ESA SP-389, 181, 1996.

Tsyganenko, N. A.: Modeling of twisted/warped magnetospheric configurations using the general deformation method, J. Geophys. Res., 103, 23 551, 1998.

Yermolaev, Yu. I., Fedorov, A. O., Vaisberg, O. L., Balebanov, V. M., Obod, Yu. A., Jimenez, R., Fleites, J., Llera, L., and Omelchenko, A. N.: Ion distribution dynamics near the Earth's bow shock: first measurements with the $2 \mathrm{D}$ ion energy spectrometer CORALL on the Interball/Tail-probe satellite, Ann. Geophysicae, 15, 533, 1997. 